\title{
MOMENTS OF ADDITIVE FUNCTIONS AND THE SEQUENCE OF SHIFTED PRIMES
}

\author{
Krishnaswami Alladi \\ Dedicated to the memory of Ernst Straus, my teacher
}

\begin{abstract}
Recently, by means of a new method involving the combinatorial sieve and the bilateral Laplace transform, we estimated asymptotically the moments of additive functions $f(n)$ for integers $n$ belonging to certain sets $S$. From such estimates the limiting distribution function of these $f(n)$, for $n \in S$, can be determined. Here the method is applied to the special sequence $S_{c}=\{p+c\}$, where $p$ runs through all the primes and $c$ is an arbitrary fixed integer. Various distribution properties of the sequence $S_{c}$, such as those given by the Brun-Titchmarch inequality and Bombieri's theorem, are used. Previously Barban had established distribution results for certain $f(n)$ when $n \in S_{c}$, but it was not known (until now) under what conditions the moments could be asymptotically estimated as well.
\end{abstract}

1. Introduction, notation and main result. In a recent paper [1] I used a new method to asymptotically estimate the moments of certain additive functions $f(n)$ for integers belonging to some special sets $S$. The method is based upon the combinatorial sieve and certain properties of the bilateral Laplace transform. It therefore has advantages over some earlier approaches in two respects. First, the use of the sieve enables one to treat a fairly large class of sets $S$. Next, the bilateral Laplace transform introduces simplification in the calculation of moments. For a detailed description of the method see [1], whereas for a comparative study of this technique against a classical background see [2].

We shall now investigate the applciations of this method to the set $S_{c}$ of shifted primes given by

$$
S_{c}=\{p+c \mid p=2,3,5, \ldots, \text { primes }\},
$$

where $c$ is any fixed positive integer. Our goal is to prove Theorem 1 below. In doing so we shall come across many interesting auxiliary results. But first we need some notation.

Additive functions $f$ are arithmetical functions satisfying $f(m \cdot n)=$ $f(m)+f(n)$, whenever g.c.d. $(m, n)=1$. For simplicity we concentrate 
here on strongly additive functions which are given by

$$
f(n)=\sum_{\substack{p / n \\ p=\text { prime }}} f(p)
$$

although the method can be applied to general additive functions as well. We associate with $f$ in (1.2) and $S_{c}$ in (1.1), the series

$$
A(x)=A_{f}(x)=\sum_{p \leq x} \frac{f(p)}{p-1}, \quad B(x)=B_{f}(x)=\sum_{p \leq x} \frac{\left|f^{2}(p)\right|}{p-1}
$$

and let

$$
M(x)=\max _{p \leq x}|f(p)| .
$$

With $A$ and $B$ as above let

$$
F_{x}(v)=\frac{1}{\pi(x-c)} \sum_{\substack{n \leq x, n \in S_{c} \\ f(n)-A(x)<v \sqrt{B(x)}}} 1,
$$

where $\pi(x)$ denotes the number of primes $\leq x$.

Next we consider the class $\mathscr{C}$ of all real $f$ that satisfy

$C_{1}: \sup _{p \leq x}|f(p)| / \sqrt{B(x)} \leq b_{f}$, is bounded for all $x$.

$C_{2}$ : there exists an $\alpha \rightarrow \infty$ with $x$ such that if $y=x^{1 / \alpha}$, then

$$
\lim _{x \rightarrow \infty} \frac{B(x)}{B(y)}=1 \text {. }
$$

We are now in a position to state our result.

TheOREM 1. Let $f \in \mathscr{C}, B(x) \rightarrow \infty$ and $K$ a probability distribution such that

(1.6) $\lim _{x \rightarrow \infty} \frac{1}{B(x)} \sum_{\substack{p \leq x \\ f(p) \leq v \sqrt{B(x)}}} \frac{f^{2}(p)}{p-1}=K(v)$, weakly in $v$.

Then there exists a probability distribution $F(v)$, such that

$$
\lim _{x \rightarrow \infty} F_{x}(v)=F(v) \text {, weakly in } v .
$$

In addition

$$
\lim _{x \rightarrow \infty} \frac{1}{\pi(x-c) B(x)^{k / 2}} \sum_{\substack{n \leq x \\ n \in S_{c}}}\{f(n)-A(x)\}^{k}=M_{k}
$$

exists and is finite for $k=1,2,3, \ldots$, where $M_{k}$ are given by

$$
M_{k}=\int_{-\infty}^{\infty} v^{k} d F(v) \text {. }
$$


Results like Theorem 1 are not entirely new. In fact there is a vast literature on the distribution of additive functions among the set $\mathbf{Z}^{+}$of all positive integers; comparatively little is known concerning distribution in subsets (see Elliott [3]). However, for the set $S_{c}$ Barban actually established that when $f \in \mathscr{C},(1.6)$ was necessary and sufficient for (1.7) to hold and later the growth condition $C_{1}$ in Barban's result was removed by Barban, Levin and Vinogradov (see [3], Vol. 2, p. 27 and p. 50). Their technique involved the probabilistic method due to Kubilius and in particular the theory of infinitely divisible distributions. However such an approach yielded no information about the higher moments. In particular it was not known whether the moments of $f$ could be asymptotically estimated as well and this we now confirm under the hypothesis of Theorem 1. In view of the above remarks, we shall, in the proof of Theorem 1 only show that (1.8) and (1.9) follow from (1.7). We suspect that $C_{1}$ is necessary for (1.8) and feel that it might be worthwhile to pursue this in detail later.

Previously, for $S_{c}$, such estimates for moments were available only in the special case when $F$ is the Gaussian distribution

$$
G(v)=\frac{1}{\sqrt{2 \pi}} \int_{-\infty}^{v} e^{-u^{2} / 2} d u .
$$

This was due to Halberstam [5] who employed a different method which was elementary and involved complicated calculations arising out of the expansion of the sum in (1.8). Our derivation of (1.8) is by a new method which involves the sieve and the bilateral Laplace transform. Our method is based upon a technique due to Elliott [4] who neatly obtained uniform upper bounds for the higher moments of additive functions $f(n)$ when $n \in \mathbf{Z}^{+}$. We noticed in [1] that incorporating sieve ideas leads to an improved method which is what we employ here.

In $\S \S 2$ and 3 we recall from [1] the main ideas of our method and also establish certain auxiliary results for $S_{c}$. Theorem 1 is proved in $\S 4$. In $\$ 5$ the special case of Theorem 1 for functions $f \geq 0$ is briefly discussed since here our method also yields a new proof of (1.7). Finally in $\S 6$, Theorem 2 , an analogue of Theorem 1 for the case $B(x) \rightarrow b<\infty$, is stated without proof.

All notation introduced so far will be retained. For instance $p$ shall always denote a prime and $f$ a strongly additive function. Also the ' $O$ ' and $\ll$ notation are equivalent and will be used interchangeably as is convenient. Implicit constants are absolute unless otherwise indicated. We shall denote by $g$, a strongly multiplicative function, namely, one that satisfies 
$g(n)=\Pi_{p \mid n} g(p)$. The truncations $f_{y}(n)$ and $g_{y}(n)$ of $f$ and $g$ at $y$ are defined by

$$
f_{y}(n)=\sum_{\substack{p \mid n \\ p \leq y}} f(p) \text { and } g_{y}(n)=\prod_{\substack{p \mid n \\ p \leq y}} g(p) .
$$

Distributions such as $F$ which arise in a weak limit form will be referred to as limiting distributions.

I would like to thank Professors P. Erdös and J. Vaaler and Dr. A. Ghosh for several discussions concerning Lemma 3. Also previously I had proved Lemma 2 only in the case when the limiting distribution $\varphi$ was continuous and it was Professor Elliott who pointed out to me that this continuity assumption could be dropped; consequently that condition does not appear in (1.8) and (1.9). Finally, I enjoyed the hospitality of the University of Texas at Austin for the academic year 1982-83 when the major portion of this work was done.

2. Two Tauberian lemmas. We begin with

LEMMA 1. Let $\varphi_{x}(v)$ be a sequence of probability distributions and $R>0$ such that

$$
\int_{-\infty}^{\infty} e^{u v} d \varphi_{x}(v) \ll \quad \text { for }|u| \leq R
$$

Then

$$
\int_{-\infty}^{\infty} v^{k} d \varphi_{x}(v) \ll \frac{k !}{R^{k}} \quad \text { for } k=1,2,3, \ldots
$$

If in addition to (2.1) we have

(2.3) $\lim _{x \rightarrow \infty} \int_{-\infty}^{\infty} e^{u v} d \varphi_{x}(v)=\ell(u)$, uniformly for $-R \leq u \leq 0$,

where $\ell(u)$ is finite, then

$$
\lim _{x \rightarrow \infty} \int_{-\infty}^{\infty} v^{k} d \varphi_{x}(v)=\mu_{k} \text { for } k=1,2,3, \ldots
$$

exists and is finite. Moreover there is a probability distribution $\varphi(v)$ such that

$$
\lim _{x \rightarrow \infty} \varphi_{x}(v)=\varphi(v) \text { weakly in } v
$$

and

$$
\mu_{k}=\int_{-\infty}^{\infty} v^{k} d \varphi(v) \text { for } k=1,2,3, \ldots
$$


For a proof of Lemma 1 see [1].

There are instances where one can asymptotically estimate the moments after initially bounding them suitably. A result along these lines is

LEMMA 2. Let $\varphi_{x}(v)$ be a sequence of probability distributions converging weakly to a limiting distribution $\varphi(v)$ as $x \rightarrow \infty$. Suppose also that for all $x$

$$
\int_{-\infty}^{\infty} v^{k} d \varphi_{x}(v) \ll_{k} 1, \text { for } k=1,2,3, \ldots
$$

Then

$$
\lim _{x \rightarrow \infty} \int_{-\infty}^{\infty} v^{k} d \varphi_{x}(v)=\int_{-\infty}^{\infty} v^{k} d \varphi(v), \text { for } k=1,2,3, \ldots
$$

Proof. First observe that (2.7) yields

$$
\int_{|v| \leq T}|v|^{k} d \varphi_{x}(v) \leq \frac{1}{T^{k}} \int_{|v| \geq T}\left|v^{2 k}\right| d \varphi_{x}(v) \ll_{k} T^{-k},
$$

$$
x, T, k \geq 1,
$$

and the same inequalities hold for $\varphi(v)$ also.

Next, let

$$
\varphi_{x}(v)=\varphi(v)+e_{x}(v)
$$

and choose $T_{1}, T_{2}>1$ such that $-T_{1}$ and $T_{2}$ are any pair of continuity points of $\varphi(v)$. So by (2.9) and (2.10) we have

$$
\begin{aligned}
\int_{-\infty}^{\infty} v^{k} d \varphi_{x}(v)=\int_{-T_{1}}^{T_{2}} v^{k} d \varphi_{x}(v)+O_{k}\left(T_{1}^{-k}+T_{2}^{-k}\right) \\
=\int_{-T_{1}}^{T_{2}} v^{k} d \varphi(v)+\int_{-T_{1}}^{T_{2}} v^{k} d e_{x}(v)+O_{k}\left(T_{1}^{-k}+T_{2}^{-k}\right) \\
=\int_{-\infty}^{\infty} v^{k} d \varphi(v)-\int_{-T_{1}}^{T_{2}} e_{x}(v) k v^{k-1} d v+T_{2}^{k} e_{x}\left(T_{2}\right) \\
\quad-\left(-T_{1}\right)^{k} e_{x}\left(-T_{1}\right)+O\left(T_{1}^{-k}+T_{2}^{-k}\right) .
\end{aligned}
$$

Since $e_{x}(v) \rightarrow 0$ almost surely in $v$, we see from (2.11) and by our choice of $T_{1}$ and $T_{2}$ that

$$
\varlimsup_{x \rightarrow \infty} \int_{-\infty}^{\infty} v^{k} d \varphi_{x}(v) \leq \int_{-\infty}^{\infty} v^{k} d \varphi(v)+O_{k}\left(T_{1}^{-k}+T_{2}^{-k}\right)
$$

with a similar lower bound for lim. Since $T_{1}, T_{2}>1$ could be arbitrarily large we get Lemma 2 from (2.12). 
3. Bounds for sums of multiplicative functions. For the purpose of treating the bilateral Laplace transform of $F_{x}(v)$ in the sequel we shall require estimates for the sum

$$
\sum_{\substack{n \leq x \\ n \in S_{\iota}}} g(n)
$$

when $0 \leq g(p) \leq 1$ for all $p$ and when $1 \leq g(p) \leq 2$ for all $p$. We begin with

Case $1.0 \leq g(p) \leq 1$.

Let $S$ be a set of integers and define

$$
S^{(d)}(x)=\sum_{\substack{s \in S, s \leq x \\ s=0(\bmod d)}} 1 .
$$

Assume that

$$
S^{(d)}(x)=\frac{S^{(1)}(x) \omega(d)}{d}+R^{(d)}(x),
$$

where $\omega$ is multiplicative, $0 \leq \omega(p) \ll 1$ for all $p$, and the 'average' of $R^{(d)}(x)$ is small in the following sense: There exists $\beta \in(0,1)$ such that for every $b>0$ there is $a>0$ satisfying

$$
\sum_{d \leq X^{\beta} / \log ^{a} X}\left|R^{(d)}(x)\right| \ll_{b} \frac{X}{\log ^{b} X},
$$

where $X=S^{(1)}(x)$. In addition assume that there is $a_{0} \geq 1$ such that

$$
\left|R^{(d)}(x)\right| \ll\left(\frac{X \log X}{d}+1\right) \cdot a_{0}^{\nu(d)},
$$

where $\nu(d)=\sum_{p \mid d} 1$. For such a set $S$ it was shown in [1] that

$$
\sum_{\substack{n \leq x \\ n \in S}} g_{y}(n)=X \prod_{p \leq y}\left(1-\frac{\omega(p)(1-g(p))}{p}\right)\{1+\eta(x, y)\},
$$

where $\eta(x, y)$ which is bounded for all $x, y \geq 1$, tends to zero as $\alpha=(\log x / \log y) \rightarrow \infty$. The proof of (3.3) makes use of ideas underlying the combinatorial sieve. In particular (3.3) implies that

$$
\sum_{\substack{n \leq x \\ n \in S}} g(n) \ll X \prod_{p \leq x}\left(1-\frac{\omega(p)(1-g(p))}{p}\right)
$$

uniformly for all $g$ satisfying $0 \leq g \leq 1$.

We apply (3.4) with $S=S_{c}$. Then $X=\pi(x-c), \omega(p)=p /(p-1)$ and (3.1) with $\beta=1 / 2$ follows from a celebrated theorem of Bombieri 
(see Halberstam and Richert [6], p. 111). Clearly (3.2) holds since $X \gg$ $x / \log x$. Thus we have

$$
\begin{aligned}
& \sum_{\substack{n \leq x \\
n \in S_{c}}} g(n) \ll \pi(x-c) \prod_{p \leq x}\left(1-\frac{1-g(p)}{p-1}\right), \\
& \text { uniformly for } 0 \leq g \leq 1 .
\end{aligned}
$$

This ends our present discussion of Case 1. We move into

Case $2.1 \leq g(p) \leq 2$.

For this we need

LEMMA 3. Let $h$ be a multiplicative function satisfying $0 \leq h(p) \leq 1$ for all $p$. Then for all square-free $n$ we have

$$
\sum_{\substack{\left.d\right|_{n} \\ d \leq \sqrt{n}}} h(d)-\sum_{\substack{\left.d\right|_{n} \\ d>\sqrt{n}}} h(d) \geq 0
$$

Proof. (Heath-Brown.) For $h$ in Lemma 3 let $D_{h}(n)$ denote the left-hand side of (3.6). First we note that if $h^{*}$ is a special multiplicative function satisfying

$$
h^{*}(p)= \begin{cases}1 & \text { if } p \in \mathscr{P}, \\ 0 & \text { if } p \notin \mathscr{P},\end{cases}
$$

then $D_{h^{*}}(n) \geq 0$ for all square-free $n$. For by setting $N=\prod_{p \in \mathscr{P}, p \mid n} p$ we get

$$
D_{h^{*}}(n)=\sum_{\substack{d \mid N \\ d \leq \sqrt{n}}} 1-\sum_{\substack{d \mid N \\ d>\sqrt{n}}} 1 \geq \sum_{\substack{d \mid N \\ d \leq \sqrt{N}}} 1-\sum_{\substack{d \mid N \\ d>\sqrt{N}}} 1 \geq 0,
$$

and that proves Lemma 3 for such $h^{*}$.

More generally, suppose $n=p_{1} \cdots p_{r}$, where $p_{i}$ are distinct primes and let $h\left(p_{i}\right)=x_{\imath} \in[0,1]$, for $i=1,2, \ldots, r$. For each $i$ we can consider $D_{h}(n)$ as a linear form in $x_{i}$ and also $D_{h}(n)=L\left(x_{1}, \ldots, x_{n}\right)$. Hence, thinking of $x_{i}$ as the first 'variable', we have for a prescribed function $h$, the inequality

$$
D_{h}(n) \geq \min _{0 \leq x_{1} \leq 1} L\left(x_{1}, \ldots, x_{r}\right) .
$$

This minimum is attained when $x_{1}$ is either 0 or 1 -say when $x_{1}=e_{1}$. Iterating this procedure we get

$$
\begin{aligned}
D_{h}(n) & \geq L\left(e_{1}, x_{2}, \ldots, x_{r}\right) \geq \min _{0 \leq x_{2} \leq 1} L\left(e_{1}, x_{2}, \ldots, x_{r}\right) \\
& =L\left(e_{1}, e_{2}, x_{2}, \ldots, x_{r}\right) \geq \cdots \geq \min _{0 \leq x_{r} \leq 1} L\left(e_{1}, \ldots, e_{r-1}, x_{r}\right) \\
& =L\left(e_{1}, e_{2}, \ldots, e_{r}\right)
\end{aligned}
$$


where $e_{i}=0$ or 1 for $i=1,2, \ldots, r$. Note that $L\left(e_{1}, \ldots, e_{r}\right)$ is equal in value to $D_{h^{*}}(n)$ for some $h^{*}$ (satisfying (3.7).) Hence (3.8) and (3.9) show that $D_{h}(n) \geq 0$ in general and that proves Lemma 3 .

We apply Lemma 3 to functions $g$ of Case 2. For such $g$ we define a multiplicative function $h$ by

$$
\sum_{\left.d\right|_{n}} h(d)=g(n) .
$$

and note that $0 \leq h \leq 1$. So by Lemma 3 we have

$$
g(n) \leq 2 \sum_{\substack{d \mid n, d \leq \sqrt{n}}} h(d)
$$

and therefore

$$
\sum_{\substack{n \leq x \\ n \in S_{c}}} g(n) \leq 2 \sum_{d \leq \sqrt{x}} h(d) \pi(x,-c, d),
$$

where

$$
\pi(x,-c, d)=\sum_{\substack{n \leq x, n \in S_{d} \\ n \equiv 0(\bmod d)}}=\sum_{\substack{p \leq x \\ p \equiv-c(\bmod d)}} 1 .
$$

By the Brun-Titchmarch inequality (see [6], p. 107) we know that

$$
\pi(x,-c, c) \ll \frac{\pi(x)}{\varphi(d)}, \quad \text { for } 1 \leq d \leq \sqrt{x},
$$

where $\varphi$ is Euler's function. Hence from (3.11) and (3.12) we get

$$
\begin{aligned}
\sum_{\substack{n \leq x \\
n \in S_{c}}} g(n) & \ll \pi(x) \sum_{d \leq \sqrt{x}} \frac{h(d)}{\varphi(d)} \\
& \ll \pi(x-c) \prod_{p \leq x}\left(1-\frac{1-g(p)}{p-1}\right)
\end{aligned}
$$

uniformly for all $g$ in Case 2, since $h(p)=g(p)-1$ and $h\left(p^{e}\right)=0$ for all $p, e \geq 2$.

REMARKS. There are other ways of proving Lemma 3.

Recently, I conjectured that for each square-free $n$ there is a mapping $m$ from the set of divisors $d$ of $n$ which are $\leq \sqrt{n}$ into the set of divisors $d^{\prime}$ of $n$ which are $>\sqrt{n}$ such that

$$
m(d)=d^{\prime} \equiv 0 \quad(\bmod d) .
$$


If (3.14) is true then we have $h\left(d^{\prime}\right) \leq h(d)$ and so Lemma 3 follows. Very recently, Erdös and Vaaler showed that more is true. By induction on $\nu(n)$, the number of prime divisors of $n$, they constructed a mapping $m_{t}$ for every $t \leq \sqrt{n}$ which takes those divisors $d$ of $n$ which are $\leq t$ into those divisors $d^{\prime}$ of $n$ that are $\geq n / t$ such that

$$
m_{t}(d)=d^{\prime} \equiv 0 \quad(\bmod d) .
$$

In particular (3.15) with $t=\sqrt{n}$ is (3.14) which yields Lemma 3. To describe this construction is complicated and hence we presented HeathBrown's argument instead.

Motivated by certain distribution properties of the divisor function $\tau(n)$, Hall and Tenenbaum have derived the following inequality [7]. Suppose $H \geq 0$ is a multiplicative function such that for each $p$, the sequence $H\left(p^{e}\right), e=0,1,2, \ldots$, is decreasing. Then

$$
\frac{\sum_{d \mid n, d \leq t} H(d)}{\sum_{d \mid n} H(d)} \geq \frac{\sum_{d \mid n, d \leq t} 1}{\tau(n)}
$$

In particular with $h$ and $n$ as in Lemma 3, (3.10) follows from (3.16) proving Lemma 3. The proof of (3.16) proceeds by induction on $\nu(n)$. We note here that (3.16) can also be proved by following Heath-Brown's idea of considering such inequalities in terms of the extrema of suitable linear forms.

4. Proof of Theorem 1. As was mentioned in $\S 1$ we will only show that (1.8) and (1.9) follow from (1.7). In view of Lemma 2 we note that it suffices to bound the moments suitably. With this in mind we first establish the following bound of a fairly general sort.

LEMMA 4. For a given real valued $f$, let $\psi(x)$ satisfy

$$
M(x)+\sqrt{B(x)} \ll \psi(x)
$$

Then for $k=1,2,3, \ldots$, we have

$$
\sum_{\substack{n \leq X_{j} \\ n \in S_{c}}}|f(n)-A(x)|^{k} \ll_{k} \pi(x-c) \psi(x)^{k},
$$

where the implicit constant in (4.2) is independent of $x$ and depends only on $k$ and on the implicit constant in (4.1). 
Proof. First we assume that $f \geq 0$ and set

$$
\varphi_{x}(v)=\frac{1}{\pi(x-c)} \sum_{\substack{n \leq x, n \in S_{c} \\ f(n)-A(x)<v \psi(x)}} 1 .
$$

Then for real $u$

(4.4) $T_{u}(x)=\int_{-\infty}^{\infty} e^{u v} d \varphi_{x}(v)=\frac{e^{-u A(x) / \psi(x)}}{\pi(x-c)} \sum_{\substack{n \leq x \\ n \in S_{c}}} g(n)$,

where

$$
g(n)=e^{u f(n) / \psi(x)} .
$$

When $u \leq 0$, we have Case 1, namely, $0 \leq g \leq 1$. Since $\psi \gg M_{f}$ by (4.1), we see that $R>0$ can be chosen, such that for $0<u \leq R$ we have $1 \leq g(p) \leq 3 / 2$; so this falls under Case 2. With such an $R$ we deduce from (4.5), (3.5) and (3.13)

(4.6) $T_{u}(x) \ll e^{-u A(x) / \psi(x)} \prod_{p \leq x}\left(1+\frac{e^{u f(p) / \psi(x)}-1}{p-1}\right)$

$$
\text { uniformly for }|u| \leq R \text {. }
$$

We have, in fact by our choice of $R$

$$
0 \leq \frac{|g(p)-1|}{p-1} \leq \frac{1}{2} \quad \text { for all } p \text { and }|u| \leq R .
$$

We make use of

$$
\log (1+t)=t+O\left(t^{2}\right), \quad|t| \leq 1 / 2 .
$$

Hence (4.5) and (4.8) yield

$$
\begin{aligned}
\log \{ & \left.\prod_{p \leq x}\left(1+\frac{g(p)-1}{p-1}\right)\right\} \\
& =\sum_{p \leq x} \frac{e^{u f(p) / \psi(x)}-1}{p-1}+O\left(\sum_{p \leq x} \frac{\left(e^{u f(p) / \psi(x)}-1\right)^{2}}{(p-1)^{2}}\right) \\
& =\sum_{1}+O\left(\sum_{2}\right), \quad|u| \leq R .
\end{aligned}
$$

Clearly from (4.1) we get

$$
\sum_{2} \ll \sum_{p \leq x} \frac{1}{p^{2}} \ll 1
$$


On the other hand from (4.1) and (4.9) we see that

$$
\begin{aligned}
\sum_{1} & =\sum_{p \leq x} \sum_{k=1}^{\infty} \frac{u^{k} f(p)^{k}}{k ! \psi(x)^{k}(p-1)} \\
& =\sum_{k=1}^{\infty} \frac{1}{k !}\left(\sum_{p \leq x} \frac{u^{k} f(p)^{k}}{\psi(x)^{k}(p-1)}\right) \\
& =\frac{u A(x)}{\psi(x)}+\sum_{k=2}^{\infty} \frac{u^{k}}{k !} \cdot O\left(\frac{B(x)}{\psi(x)^{2}} \frac{M(x)^{k-2}}{\psi(x)^{k-2}}\right) \\
& =\frac{u A(x)}{\psi(x)}+o(1), \text { for }|u| \leq R .
\end{aligned}
$$

Thus by putting (4.6), (4.9), (4.10) and (4.11) together, we deduce that

$$
T_{u}(x) \ll 1, \text { uniformly for }|u| \leq R \text {. }
$$

So (4.2) for $f \geq 0$ is a consequence of (4.3), (4.12) and (2.2) of Lemma 1.

In general a real valued $f$ can be decomposed into $f=f^{+}-f^{-}$, where $f^{+}$and $f^{-}$are strongly additive functions generated by

$$
f^{+}(p)=\max (0, f(p)), f^{-}(p)=-\min (0, f(p)) .
$$

Note that if (4.1) holds for $f$, then we also have

$$
m_{f^{ \pm}}(x)+B_{f^{ \pm}}(x) \ll \psi(x) .
$$

So from the truth of (4.2) for non-negative $f$ and (4.13) we get

$$
\sum_{\substack{n \leq x \\ n \in S_{\iota}}}\left|f^{ \pm}(n)-A_{f^{ \pm}}(x)\right| \ll_{k} \pi(x-c) \psi(x)^{k}, \quad k=1,2,3 \ldots
$$

Finally observe that

$$
|a-b|^{k} \ll_{k}|a|^{k}+|b|^{k}, \quad k=1,2,3, \ldots
$$

holds for all real $a$ and $b$. So by decomposing

$$
f(n)-A(x)=\left\{f^{+}(n)-A_{f^{+}}(x)\right\}-\left\{f^{-}(n)-A_{f^{-}}(x)\right\}
$$

we get Lemma 4 from (4.14) and (4.15).

Proof of Theorem 1. Let $f \in \mathscr{C}$ satisfy (1.6). Take $\psi(x)=\sqrt{B(x)}$ in which case $\varphi_{x}(v)$ in (4.3) is $F_{x}(v)$. We identify (4.2) with (2.7) of Lemma 2. So by (1.7) and Lemma 2 we see that (1.8) holds. Finally (1.9) is a consequence of (1.7) and (1.8) since the moments $M_{k}$, by Lemma 1, are $\ll k ! / R^{k}$ in size. Theorem 1 is proved. 
5. Theorem 1 for $f \geq 0$. In proving (1.8) for real valued $f$ we made use of (1.7). Our method initially supplied only upper bounds for the moments of such $f$. So in order to obtain asymptotic estimates for the moments from such bounds we had to use (1.7). If asymptotic estimates for the moments could be obtained directly, then the existence of a limiting distribution would follow, provided the moments do not grow too rapidly. Such asymptotic estimates are possible by our method when $f \geq 0$ and so in this case we do not require Kubilius' method to derive (1.7). Since this proof of Theorem 1 for $f \geq 0$ contains certain new features we sketch the main ideas briefly. Let

$$
F_{x, y}(v)=\frac{1}{\pi(x-c)} \sum_{\substack{n \leq x \\ f_{v}(n)-A(y)<v \sqrt{B(y)}}} 1 .
$$

First we note that for $f \geq 0,(3.3)$ provides an asymptotic estimate for $T_{u}(x, y)$, the bilateral Laplace transform of $F_{x, y}(v)$ when $u \leq 0$, provided $\alpha \rightarrow \infty$ with $x$. On the other hand (3.13) provides an upper bound for $T_{u}(x, y)$ when $0 \leq u \leq R$. A more careful analysis of $\Sigma_{1}$ and $\Sigma_{2}$ shows that $\Sigma_{2} \rightarrow 0$ as $B(x) \rightarrow \infty$, and

$$
\Sigma_{1} \rightarrow \int_{-\infty}^{\infty} \frac{e^{u v}-1-u v}{v^{2}} d K(v), \quad \text { uniformly for }-R \leq u \leq 0 .
$$

So when $\alpha=\alpha(x)$ (a fixed function of $x$ ) tends to infinity with $x$, we have

$$
\begin{aligned}
& T_{u}(x, y) \rightarrow \ell(u)=\exp \left\{\int_{-\infty}^{\infty} \frac{e^{u v}-1-u v}{v^{2}} d K(v)\right\}, \\
& \text { uniformly for }-R \leq u \leq 0,
\end{aligned}
$$

whereas

$$
T_{u}(x, y) \ll 1, \quad \text { for } 0 \leq u \leq R .
$$

Set $\varphi_{x}(v)=F_{x, v}(v)$ in Lemma 1 and compare (5.2) and (5.3) with (2.1) and (2.3). Theorem 1 for $f_{y}(n)$ now follows provided $f \geq 0$ and $\alpha \rightarrow \infty$.

In order to bridge the gap between $f_{y}(n)$ and $f(n)$ we utilise the following lemma (for a proof see Elliott [3], Vol. 1., p. 173).

LEMMA 5. Let $\beta<2$ and $f^{*}(n)$ be any real valued strongly additive function. Then

$$
\sum_{\substack{n \leq x \\ n \in S_{\iota}}}\left|f^{*}(n)-A_{f^{*}}(x)\right|^{\beta} \ll_{\beta} \pi(x-c) B_{f^{*}}(x)^{\beta / 2}
$$

holds uniformly for all such $f^{*}$. 
We set $f^{*}=f-f_{y}$ in Lemma 5. By the Cauchy-Schwarz inequality $A(x)-A(y) \leq \sqrt{\log \alpha \cdot(B(x)-B(y))}$. Choose $\alpha \rightarrow \infty$ such that $C_{2}$ holds and $A(x)-A(y)=o(\sqrt{(B(x)-B(y))})$. This shows that the weak limit of $F_{x, y}(v)$ is the weak limit of $F_{x}(v)$ and so (1.7) is established for $f(n) \geq 0$.

Consider the decomposition

$$
\begin{aligned}
f(n)-A(x)= & \left\{f_{y}(n)-A(y)\right\}+\left\{f(n)-f_{y}(n)\right\} \\
& +\{A(x)-A(y)\} .
\end{aligned}
$$

In order to estimate the sum on the left side of (1.8) we use (4.15). The contribution due to $A(x)-A(y)$ is negligible by our choice of $\alpha$. From the multinomial expansion and growth conditions $C_{1}$ and $C_{2}$ the contribution due to $f(n)-f_{y}(n)$ can be bounded by $O\left(\pi(x-c) B(x)^{k / 2}\right)$. Finally the sum involving $f_{y}(n)-A(y)$ can be bounded suitably by appealing to the truth of (1.8) for $f_{y}$. Hence ultimately we get (4.2) with $\psi(x)$ replaced by $\sqrt{B(x)}$. As before, (1.8) for $f \geq 0$ now follows from this bound and (1.7). That proves Theorem 1 for non-negative $f \in \mathscr{C}$.

REMARKs. The moments $M_{k}$ (as was noticed earlier) are $\ll k ! / R^{k}$. The analysis underlying (6.2) along with this bound for $M_{k}$ shows that

$$
\ell(z) \stackrel{\text { def }}{=} \exp \left\{\int_{-\infty}^{\infty} \frac{e^{z v}-1-z v}{v^{2}} d K(v)\right\}=\int_{-\infty}^{\infty} e^{z v} d F(v)
$$

is analytic in $|z|<R$. Therefore $\ell(z)$ is the analytic characteristic function of $F$. Therefore the moments $M_{k}$ in in (1.8) can be calculated from $\ell(z)$ by using the relation

$$
M_{k}=\left.\frac{d^{k} \ell(z)}{d z^{k}}\right|_{z=0} .
$$

The appeal to $f_{y}(n)$ in the proof presented here is a limitation in our method because the Sieve asymptotic estimate (3.3) is true only when $\alpha \rightarrow \infty$ with $x$. However this limitation is not harsher than in earlier approaches, all of which utilised such Sieve estimates in one way or other and so required the introduction of the truncated function $f_{y}(n)$. In fact its the use of the truncation which accounts for the dependence on $C_{2}$. It might appear to the reader that in our proof of Theorem 1 presented in $\S 4$, the truncation $f_{y}(n)$ was not used. But it should be noted that we utilised the truth of (1.7), due to Barban, and his proof of (1.7) was by the Kubilius method which also employs $f_{y}(n)$. 
6. An analogue of Theorem 1. There is an analogue of Theorem 1, in the case $B(x) \rightarrow b<\infty$, namely

THEOREM 2. Let $f(p)=O(1)$ for all $p$ and $B(x) \rightarrow b<\infty$. Then there is a probability distribution $F^{*}(v)$ such that

$$
\lim _{x \rightarrow \infty} \frac{1}{\pi(x-c)} \sum_{\substack{n \leq x, n \in S_{c} \\ f(n)-A(x)<v}} 1=F^{*}(v), \text { weakly in } v .
$$

In addition

$$
\lim _{x \rightarrow \infty} \frac{1}{\pi(x-c)} \sum_{n \leq x, n \in S_{c}}\{f(n)-A(x)\}^{k}=M_{k}^{*}
$$

exists and is finite for $k=1,2,3, \ldots$, where

$$
M_{k}^{*}=\int_{-\infty}^{\infty} v^{k} d F^{*}(v)
$$

Finally,

$$
\ell^{*}(z)=\prod_{p}\left(1-\frac{e^{z f(p)}}{p-1}\right) e^{-z f(p) /(p-1)}
$$

is the analytic characteristic function of $F^{*}$ around the origin and therefore

$$
M_{k}^{*}=\left.\frac{d^{k} \ell^{*}(z)}{d z^{k}}\right|_{z=0}, \text { for } k=1,2,3, \ldots
$$

Since $B(x) \rightarrow b<\infty$, condition $C_{2}$ is redundant and so the details are simpler. Hence we omit the proof of Theorem 2. However, we wish to mention here, that similar to Theorem $1,(6.1)$ can be proved by the probabilistic method of Kubilius. After using our method to bound the $k$ th moments suitably, (6.2) and (6.3) can be deduced from (6.1). If one is interested only in $f \geq 0$, then analogous to the arguments of $\S 5,(6.1)$ also can be established by our method.

\section{REFERENCES}

[1] K. Alladi, A Study of the Moments of Additive Functions Using Laplace Transforms and Sieve Methods, Proc. Conf. on Number Theory, Ootacamund, India (1984), Springer Lecture Notes (to appear).

[2] Moments of Additive Functions and Sieve Methods, in Proc. New York Number Theory Seminar (1982), Springer-Lecture Notes, Vol. 1051, 1-25.

[3] P. D. T. A. Elliott, Probabilistic Number Theory, Vols. 1 and 2, Grundlehren 239 and 249, Springer-Verlag, Berlin New York (1980). 
[4] High power analogues of the Turan-Kubilius inequality and an application to Number Theory, Canad. J. Math., 32 (1980), 893-907.

[5] H. Halberstam, On the distribution of additive number-theoretic functions III, J. London Math. Soc., 31 (1956), 14-27.

[6] H. Halberstam and H.-E. Richert, Sieve Methods, Academic Press New York (1974).

[7] R. R. Hall and G. Tenenbaum, On the local behaviour of some arithmetical functions, Acta Arith., 43 (1984), 375-390.

Received June 15, 1984. Work done at the University of Texas, Austin, while on leave of absence from MATSCIENCE, The Institute of Mathematical Sciences, Madras, India.

\section{MATSCIENCE}

The Institute of Mathematical Sciences

MADRAS - 600113 INDIA 
